\title{
Production of hydroxycinnamoyl anthranilates from glucose in Escherichia coli
}

\author{
Aymerick Eudes ${ }^{1,2+}$, Darmawi Juminaga ${ }^{1,3+}$, Edward E K Baidoo ${ }^{1}$, F William Collins ${ }^{4}$, Jay D Keasling 1,2,3,5 \\ and Dominique Loqué ${ }^{1,2^{*}}$
}

\begin{abstract}
Background: Oats contain hydroxycinnamoyl anthranilates, also named avenanthramides (Avn), which have beneficial health properties because of their antioxidant, anti-inflammatory, and antiproliferative effects. The microbial production of hydroxycinnamoyl anthranilates is an eco-friendly alternative to chemical synthesis or purification from plant sources. We recently demonstrated in yeast (Saccharomyces cerevisiae) that coexpression of 4-coumarate: CoA ligase (4CL) from Arabidopsis thaliana and hydroxycinnamoyl/benzoyl-CoA/anthranilate N-hydroxycinnamoyl/benzoyltransferase (HCBT) from Dianthus caryophyllusenabled the biological production of several cinnamoyl anthranilates upon feeding with anthranilate and various cinnamates. Using engineering strategies to overproduce anthranilate and hydroxycinnamates, we describe here an entire pathway for the microbial synthesis of two Avns from glucose in Escherichia coli.
\end{abstract}

Results: We first showed that coexpression of HCBT and Nt4CL1 from tobacco in the E. coli anthranilate-accumulating strain W3110 trpD9923 allowed the production of Avn D [N-(4'-hydroxycinnamoyl)-anthranilic acid] and Avn F [N-(3',4'dihydroxycinnamoyl)-anthranilic acid] upon feeding with $p$-coumarate and caffeate, respectively. Moreover, additional expression in this strain of a tyrosine ammonia-lyase from Rhodotorula glutinis (RgTAL) led to the conversion of endogenous tyrosine into $p$-coumarate and resulted in the production of Avn D from glucose. Second, a 135-fold improvement in Avn D titer was achieved by boosting tyrosine production using two plasmids that express the eleven genes necessary for tyrosine synthesis from erythrose 4-phosphate and phosphoenolpyruvate. Finally, expression of either the $p$-coumarate 3-hydroxylase Sam5 from Saccharothrix espanensis or the hydroxylase complex HpaBC from E. coli resulted in the endogenous production of caffeate and biosynthesis of Avn F.

Conclusion: We established a biosynthetic pathway for the microbial production of valuable hydroxycinnamoyl anthranilates from an inexpensive carbon source. The proposed pathway will serve as a platform for further engineering toward economical and sustainable bioproduction of these pharmaceuticals and other related aromatic compounds.

Keywords: Avenanthramide, Tranilast, BAHD, Antioxidant, Anti-inflammatory, Tyrosine, Anthranilate, Hydroxycinnamate, Biological synthesis, Escherichia coli

\section{Background}

Hydroxycinnamoyl anthranilates are part of the large cinnamoyl anthranilates family, a class of molecules with beneficial health properties. For example, avenanthramides (Avns) are natural hydroxycinnamoyl anthranilates found in oats (Avena sativa L.) at low concentrations (a few parts

\footnotetext{
* Correspondence: dloque@|bl.gov

${ }^{\dagger}$ Equal contributors

'Joint BioEnergy Institute, Emeryville, CA 94608, USA

${ }^{2}$ Physical Biosciences Division, Lawrence Berkeley National Laboratory,

Berkeley, CA 94720, USA

Full list of author information is available at the end of the article
}

per million in grains) and whose antioxidant, antiinflammatory, and antiproliferative effects are considered to contribute to the health benefits of oatmeal consumption [1-6]. The antioxidant effects of Avns have been established in several animal studies [7-9], and their anti-inflammatory properties illustrated in model systems of atherosclerosis, diabetes, itching, and breast cancer. In particular, Avnenriched oat extracts, synthetic dihydroavenanthramide D (DHAvn D) and Avn $\mathrm{C}$ methyl ester $\left(\mathrm{CH}_{3}\right.$-Avn $\left.\mathrm{C}\right)$ (Figure 1A) were shown to inhibit the activation of the NF$\mathrm{kB}$ transcription factor, which is a master regulator of infection and inflammation [10-14]. Consequently, DHAvn D

\section{Biomed Central}


has been developed as a drug to reduce histamine-related skin disorders [15]. Lastly, the antiproliferative effects of Avn have been demonstrated on vascular muscle and colonic cancer cell lines [16-18]. Similarly, tranilast [N-( $3^{\prime} 4^{\prime}-$ dimethoxycinnamoyl)-anthranilic acid] (Figure 1A) is a synthetic cinnamoyl anthranilate used as an antihistamine in Japan and South Korea for the treatment of allergic disorders, hypertrophic scars, and keloids [19-21]. Tranilast has anti-inflammatory and antiproliferative effects and is currently evaluated clinically for the treatment of multiple sclerosis and various arthritic conditions [22-24]. Moreover, the antitumor potential of tranilast has been evidenced in several clinical trials [25], and the design of analogues that exhibit higher anti-fibrotic activity has been extensively investigated [26-30].

Using microbes for biological synthesis of therapeutic drugs or precursors offers an alternative production strategy to commonly employed methods such as direct extraction from source organisms or chemical synthesis. Microbial expression systems have several advantages such as reduced requirements for toxic chemicals and natural resources; consistent quality; scalability; simple extraction; and potential for higher synthesis efficiency [31,32]. Taking into consideration the expanding number of therapeutic applications for cinnamoyl anthranilates, as well as the fact that these molecules are currently synthesized chemically or extracted from food sources $[33,34]$, we attempted to design a pathway for their de novo production from glucose using E. coli as a production platform.

HCBT is an acetyltransferase from the BAHD family [35], which couples $p$-coumaroyl-CoA with anthranilate via an amide bond to produce Avn D (Figure 1B) [36]; while $4 \mathrm{CL}$ enzymes convert cinnamates into their corresponding CoA thioesters [37]. We previously engineered a yeast strain that coexpresses $4 \mathrm{CL}$ and HCBT for the production of several cinnamoyl anthranilates, such as Avn D and Avn F, upon feedings with anthranilate and various cinnamates. This highlighted the potential of using these enzymes for the biological production of cinnamoyl anthranilates [38]. E. coli is a host of choice for the expression of complex pathways and the production of elaborate molecules such as aromatic compounds from cheap carbon sources $[39,40]$.

In this study, we primarily focused on the biological synthesis of Avn D, which features a basal core structure of hydroxycinnamoyl anthranilates. For this purpose, a previously characterized $E$. coli anthranilate-accumulating strain was selected as a chassis $[41,42]$. In that strain, coexpression of Nt4CL1 and HCBT led to the production of Avn D and Avn F when the culture medium was supplemented only with $p$-coumarate and caffeate, respectively. This validated the functional expression and activity of both plant enzymes in our chassis. The production system was then affranchised from precursor feeding by additional expression of $\operatorname{RgTAL}$, which

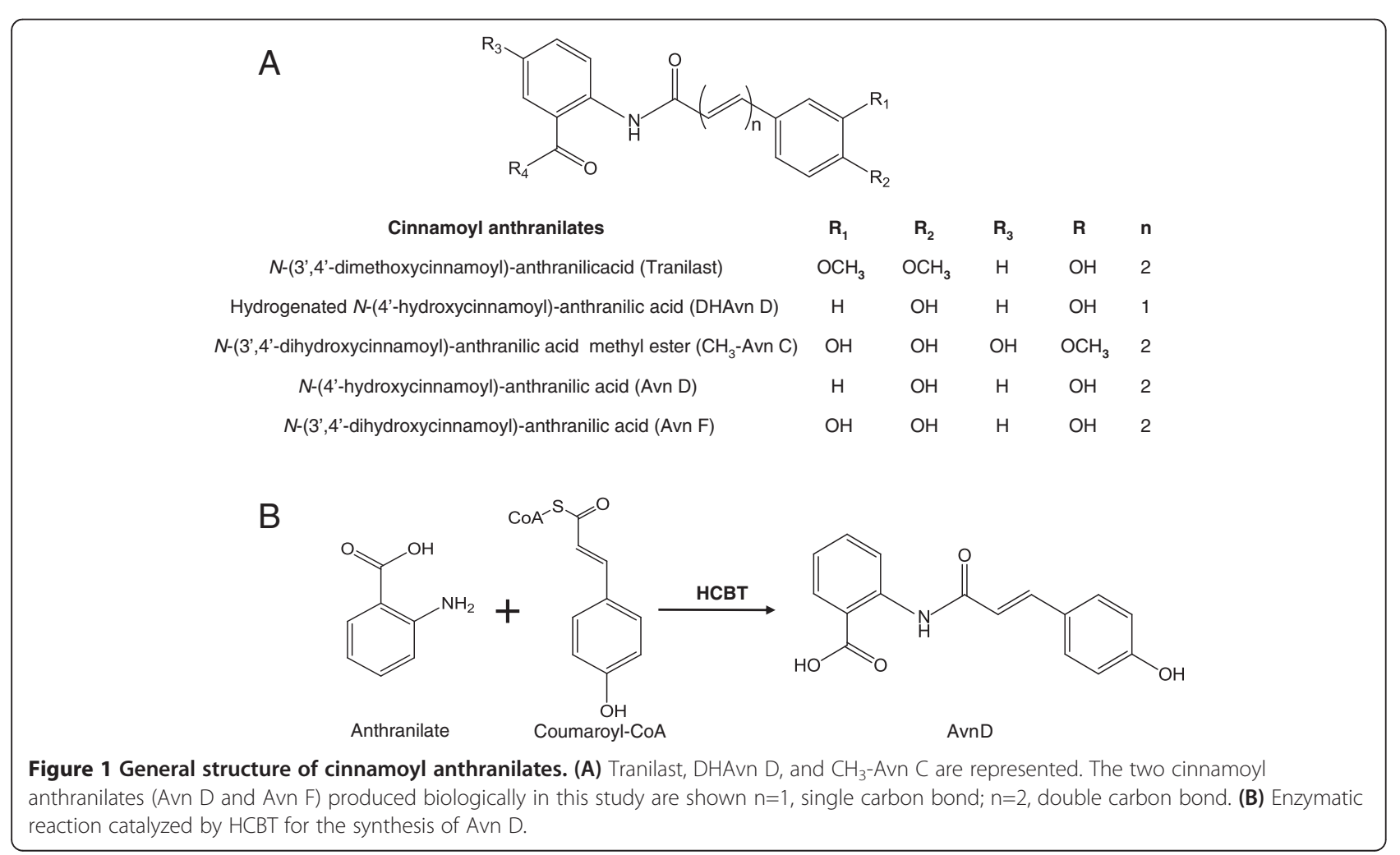


converts tyrosine into $p$-coumarate $[43,44]$ (Figure 2). Avn D biosynthesis was further enhanced by expressing a two-plasmid-based modular biosynthetic pathway for tyrosine overproduction from glucose [45]. Finally, Avn F was also biologically produced de novo upon expression of either Sam5 or HpaBC, which are two hydroxylases that convert $p$-coumarate into caffeate $[46,47]$.

\section{Results and discussion}

\section{Expression of Nt4CL1 and HCBT in E. coli strain W3110} trpD9923

E. coli W3110 trpD9923 strain is a tryptophan auxotroph that over-accumulates anthranilate due to a nonsense mutation in the $\operatorname{trpD}$ gene, which abolishes anthranilate phosphoribosyltransferase activity but does not affect anthranilate synthase activity $[41,42]$. This strain was shown to be suitable for metabolic engineering because expression of genes from the shikimate pathway further increased anthranilate production [41]. We first constructed pAvn plasmid for coexpression of Nt4CL1, which encodes a $4 \mathrm{CL}$ that converts $p$-coumarate and caffeate into their corresponding CoA thioesters [48], and HCBT. To confirm that HCBT can catalyze the condensation of coumaroylCoA with anthranilate and produce Avn D in W3110 trpD9923, the strain was transformed with pAvn and grown in the presence of $p$-coumarate as a precursor. Cultures of W3110 trpD9923 harboring an empty vector were also grown as a negative control. Only in the case of the strain expressing pAvn, LC-TOF MS analysis of the culture medium revealed a peak ( $\mathrm{Rt}=11.75 \mathrm{~min}$ ) that corresponds to Avn D by comparison with an authentic standard solution (Figure 2A). Similarly, the engineered strain produced some Avn F $(\mathrm{Rt}=10.56 \mathrm{~min})$ when $p$-coumarate was substituted by caffeate in the medium (Figure 2B). This result confirms the affinity of HCBT for caffeoyl-CoA. It also demonstrates secretion of Avn outside of the production host, because the Avn D and Avn F content inside E. coli cells represented less than $5 \%$ of the amount quantified from the medium (data not shown).

\section{Biosynthesis of Avn D from glucose and titer} improvement using a tyrosine overproduction strategy

To produce Avn D - without supplying costly precursors such as $p$-coumarate to the engineered $E$. coli strain - we designed a plasmid (pAvnD) that contains in a single operon $H C B T, N t 4 C L 1$, and a gene encoding $R g$ TAL for the conversion of tyrosine into $p$-coumarate (Figure 3A, B). Analysis of the culture medium of cells harboring pAvnD and grown for 24 hours revealed the presence of $p$ coumarate $(1.0 \pm 0.1 \mu \mathrm{M})$, which was produced from endogenous tyrosine upon $\operatorname{Rg}$ TAL activity, and a detectable amount of Avn D ( 200 nM) (Table 1). Moreover, a 15\% reduction of the final biomass density was observed for this strain compared to the control. We recently reported on a strategy for the overproduction in E. coli of tyrosine using two plasmids ( $\mathrm{pS} 0$ and $\mathrm{pY}$ ) that contain all the genes required for the synthesis of tyrosine from erythrose 4phosphate and phosphoenolpyruvate (Figure 3A, B) [45]. As expected, this strategy applied to the W3110 trpD9923 strain not only increased tyrosine titers (665-fold), but also enhanced anthranilate production (3.5-fold), since both metabolites are derived from chorismate via the shikimate pathway (Figure 3A; Table 1). This engineered strain, despite having a slower growth rate, showed no difference in final biomass density compared to that harboring empty vector controls. Furthermore, co-transformation of compatible

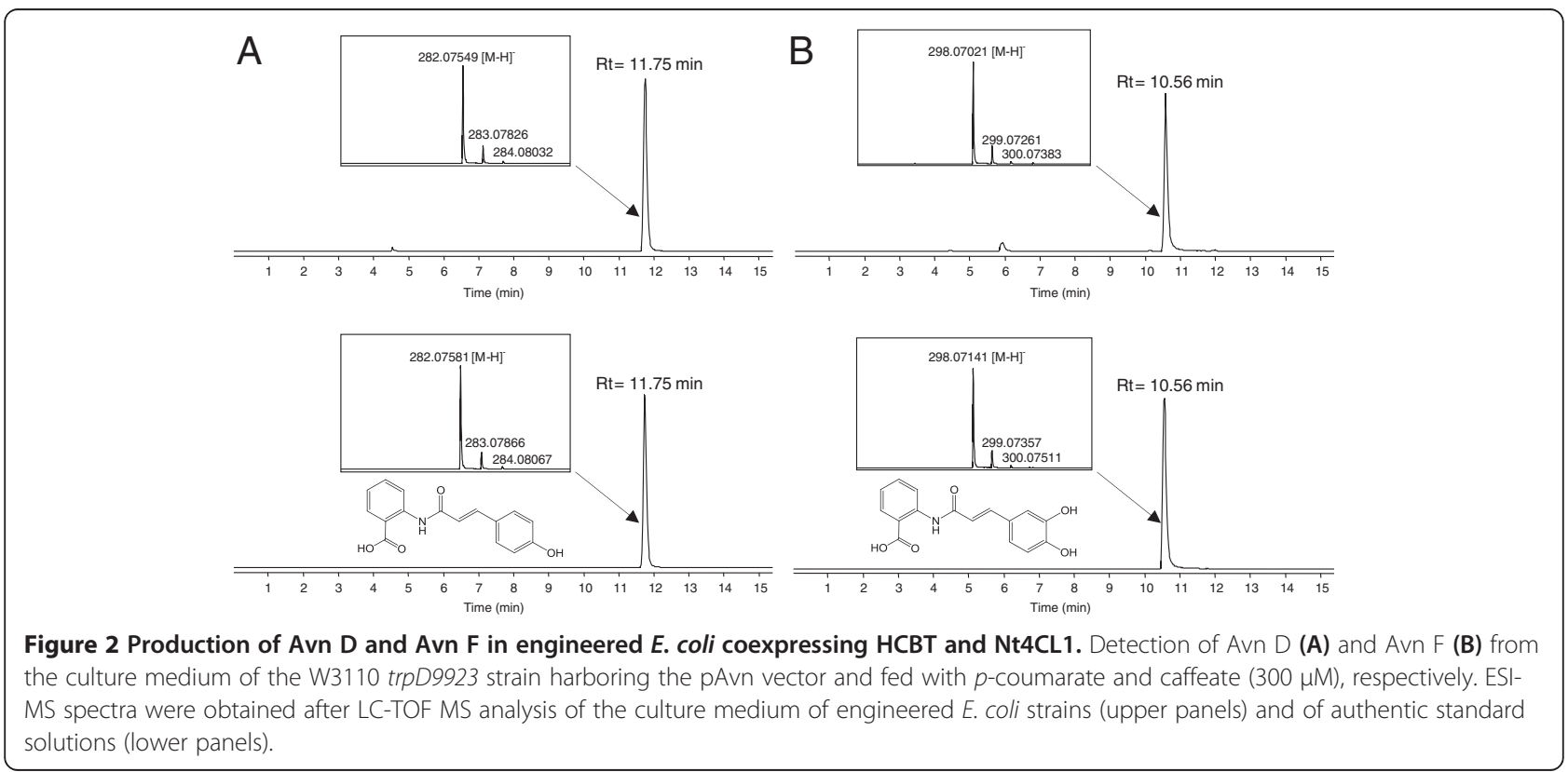




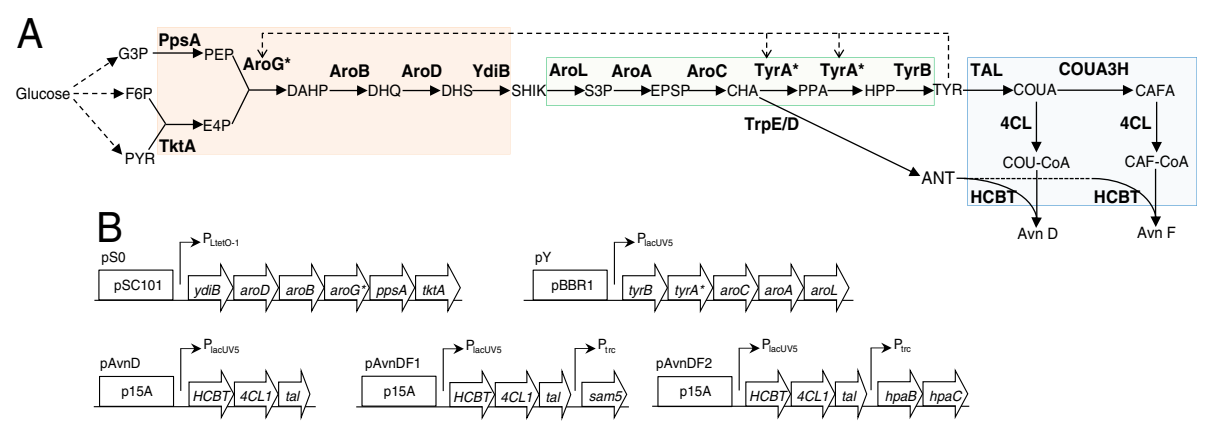

Figure 3 Biosynthetic pathways for the microbial production of hydroxycinnamoyl anthranilates from glucose. (A) Three compatible plasmids were used for the biological production of Avn D and Avn F from glucose. A first plasmid is a shikimate module which contains all the six genes for the production of shikimate (SHIK) from pyruvate (PYR), fructose 6-phosphate (F6P), and glyceraldehydes 3-phosphate (G3P) (orange box). A second plasmid is a tyrosine module which contains all the five genes for the production of tyrosine (TYR) from SHIK (green box). The third plasmid is the cinnamoyl anthranilate module which contains up to five genes for the conversion of TYR into two hydroxycinnamoyl-CoAs and their coupling to anthranilate (ANT) for the production of Avn D and Avn F (blue box).CAFA, caffeate; CAF-CoA, caffeoyl-CoA; CHA, chorismate; COUA, p-coumarate; COU-CoA, p-coumaroyl-CoA; DAHP, 3-deoxy-D-arabino-heptulosonate; DHQ, dehydroquinate; DHS, dehydroshikimate; E4P, erythrose 4-phosphate; EPSP, 5-enolpyruvoylshikimate 3-phosphate; HPP, 4 hydroxyphenlypyruvate; PEP, phosphoenolpyruvate; PPA, prephenate; and S3P, shikimate 3-phosphate. The enzymes (in bold face) are as follows: PpsA, phosphoenolpyruvate synthase; TktA, transketolase A; AroG, DAHP synthase; AroB, DHQ synthase; AroD, DHQ dehydratase; YdiB, shikimate dehydrogenase; AroL, shikimate kinase II; AroA, EPSP synthase; AroC, chorismate synthase; TrpE/D, anthranilate synthase I/Il; TyrA, chorismatemutase/prephenate dehydrogenase; and TyrB, tyrosine aminotransferase; TAL, tyrosine ammonia-lyase; 4CL, p-coumarate:CoA ligase; COUA3H, p-coumarate 3-hydroxylase; HCBT, hydroxycinnamoyl/benzoyl-CoAvanthranilate Nhydroxycinnamoyl/benzoyltransferase. The dashed lines indicate where feedback inhibitions occur. Allosteric regulation of AroG and TyrA were removed by employing their respective feedback-resistant mutants, AroG* (D146N) and TyrA* (M53l; A354V), respectively. (B) Structures of the three plasmids used for cinnamoyl anthranilates production. The open blocks indicate the origins of replication, the open arrows represent the genes, and the angled arrows indicate the promoters.

shikimate (pS0) and tyrosine (pY) plasmids with pAvnD led to a 135-fold increase in extracellular Avn D $(27.3 \pm 0.1 \mu \mathrm{M})$ compared to the production achieved using pAvnD alone, after $24 \mathrm{~h}$ of culture (Table 1). The analysis of the culture medium also revealed that $p$-coumarate content $(\sim 32 \mu \mathrm{M})$ was much lower compared to that of tyrosine $(\sim 6 \mathrm{mM})$, suggesting that $\operatorname{RgTAL}$ is a rate-limiting enzyme in the pathway (Table 1). As observed for the strain containing $\mathrm{pAvnD}$ alone, the strain harboring the three plasmids had a $15 \%$ reduction of the final biomass density.

\section{Conversion of $p$-coumarate into caffeate and production of Avn F using Sam5}

For the biological production of caffeate, and ultimately Avn F, we generated pAvnDF1 plasmid, which adds into the pAvnD backbone sam5 under the control of the trc promoter (Figure 3A, B). Sam5 is a $p$-coumarate 3hydroxylase that has been successfully expressed in $E$. coli for the biological synthesis of caffeate [49-51]. Expression of the genes harbored on pAvnDF1 plasmid in the W3110 trpD9923 strain resulted in the production of a small amount of caffeate in the culture medium, but no Avn could be detected (Table 2). However, cotransformation of pAvnDF1 with pS0 and pY not only enhanced caffeate production ( 230 -fold), but also led to the biosynthesis of Avn F ( 110 nM) in addition to Avn D (Table 2). No extracellular $p$-coumarate could be detected in these cultures, suggesting that most of it was efficiently converted into caffeate by Sam 5 .

Interestingly, LC-TOF MS analysis revealed an additional new peak in the culture medium of the strains harboring pAvnDF1 and expressing Sam5. This peak was found to correspond to 3,4,5-trihydroxycinnamate based on the mass and elution time of an authentic standard (Additional file 1: Figure S1), and the 3,4,5trihydroxycinnamate content represented $\sim 1.6 \mu \mathrm{M}$ in

Table 1 Production of Avn D and precursors by engineered W3110 trpD9923 E. coli strains

\begin{tabular}{lllll}
\hline Plasmids & Compounds $(\boldsymbol{\mu M})$ & & & \\
\cline { 2 - 5 } & Anthranilate & Tyrosine & -Coumarate & Avn D \\
\hline Empty pZS21+pBbB5a & $1646 \pm 63$ & $9.3 \pm 1.3$ & nd & nd \\
pSO +pY & $5878 \pm 311$ & $6201 \pm 598$ & nd & nd \\
pAvnD & $1564 \pm 58$ & $6.8 \pm 0.9$ & $1.0 \pm 0.1$ & $0.2 \pm 0.1$ \\
pSO + pY + pAvnD & $5780 \pm 251$ & $5963 \pm 219$ & $32.6 \pm 3.4$ & $27.3 \pm 1.4$ \\
\hline
\end{tabular}

Values are the means $\pm S D$ of five independent clones. $n d$ not detected. 
the culture medium of the pAvnDF1 containing strain and reached $48 \mu \mathrm{M}$ when the tyrosine production pathway $(\mathrm{pS} 0+\mathrm{pY})$ was co-expressed in the pAvnDF1containing strain. These observations strongly suggest that Sam5 can not only convert $p$-coumarate into caffeate, but also caffeate into 3,4,5-trihydroxycinnamate. To validate this hypothesis, an E. coli strain expressing Sam 5 alone was grown in the presence of caffeate and the culture medium analyzed for the presence of 3,4,5trihydroxycinnamate. Conclusively, this new compound was detected in the medium of the Sam 5 strain but not in that of an empty vector control strain (Figure 4).This is, to our knowledge, the first report of an enzyme capable of hydroxylating caffeate. Although the conversion of caffeate into 3,4,5-trihydroxycinnamate is not desirable for the production of Avn F (and perhaps inhibitory for Nt4CL1 activity), this novel hydroxylating property for Sam5 presents an opportunity for the enzymatic synthesis of trihydroxylated cinnamoyl anthranilates. In this regard, we previously demonstrated in yeast the widerange of substrate specificity for Arabidopsis 4CL5 and HCBT toward various substituted cinnamates and cinnamoyl-CoAs, respectively [38].

\section{Conversion of $p$-coumarate into caffeate and production of Avn $\mathrm{F}$ using the $\mathrm{HpaBC}$ complex}

The enzyme complex consisting of a 4-hydroxyphenylacetate 3-hydroxylase $(\mathrm{HpaB})$ and a flavin:NADH reductase $(\mathrm{HpaC})$ from $E$. coli was tested for the biological production of caffeate and Avn F. The operon hpaBC is involved in 4-hydroxyphenylacetate degradation and several studies showed that the HpaBC enzyme complex can accept a broad range of substrates including tyrosine and $p$-coumarate $[46,52,53]$. We constructed a pAvnDF2 plasmid by placing the $h p a B C$ operon under the control of the trc promoter into pAvnD plasmid (Figure 3B). Transformation of pAvnDF2 into E. coli W3110 trpD9923 resulted in the production of small amount of caffeate in the culture medium, but only Avn D could be detected (Table 2). By contrast, co-transformation of pAvnDF2 with $\mathrm{pS} 0$ and $\mathrm{pY}$ enhanced caffeate production ( 115-fold) and led to the biosynthesis of Avn F ( $\sim 540 \mathrm{nM})$ in addition to Avn D (Table 2). Unlike the results of the biosynthesis of Avn F using Sam5, the expression of HpaBC maintained higher Avn D titers and did not produce any 3,4,5-trihydroxycinnamate nor entirely deplete $p$-coumarate content. This suggests that $\mathrm{HpaBC}$ is less efficient than Sam5 at converting $p$-coumarate into caffeate in our system, yet nevertheless Avn F titers using $\mathrm{HpaBC}$ were $~ 5$ fold higher compared to those achieved using Sam5 (Table 2). Alternatively, the higher caffeate content and lower AvnF titers obtained using Sam5 could reflect a negative effect of 3,4,5-trihydroxycinnamate on 4CL1 activity. Moreover, we observed a reduction in tyrosine titers compared to those measured from the culturesof E. coli W3110 trpD9923 harboring $\mathrm{pAvnD}$ or $\mathrm{pAvnDF}$. This was probably due to $\mathrm{HpaBC}$ activity, which can also convert tyrosine into L-dopa. Conclusively, we found that L-dopa concentration was $\sim 4.4 \mathrm{mM}$ in the culture medium of the $\mathrm{pSO} / \mathrm{pY} /$ pAvnDF2 strain. Furthermore, based on previous studies showing that some tyrosine ammonia-lyases convert Ldopa into caffeate $[46,54]$, an $E$. coli strain that expresses $\operatorname{RgTAL}$ alone was created and grown in the presence of Ldopa. Interestingly, analysis of the culture medium of the $\operatorname{RgTAL}$ strain revealed the presence of caffeate, which was absent in the medium of an empty vector control strain (Figure 5). These results demonstrate that $\operatorname{RgTAL}$ exhibits some L-dopa ammonia-lyase activity and suggest that part of the caffeate produced in the strains harboring pAvnDF2 could be derived from L-dopa.

\section{Conclusions}

This work is an example of biological production of valuable aromatic metabolites using a tyrosine-overproducing strategy applied to an anthranilate-accumulating strain. Considering the anthranilate titers achieved with the strain containing only the shikimate and tyrosine modules, the maximum theoretical yield for Avn D in this background would be $\sim 5.8 \mathrm{mM}$. However, much lower Avn D titers were obtained for the strain harboring $\mathrm{pSO}, \mathrm{pY}$ and $\mathrm{pAvnD}$, probably due to poor conversion of tyrosine into $p$-coumarate as previously observed in various studies using heterologous expression of TALs $[46,49,50]$, and potentially to the limited intracellular pools of coenzyme A availability [55]. It is particularly noteworthy that, because of its specificity to anthranilate as an acceptor, the BAHD

Table 2 Production of Avn F and precursors by engineered W3110 trpD9923 E. coli strains

\begin{tabular}{|c|c|c|c|c|c|c|}
\hline \multirow[t]{2}{*}{ Plasmids } & \multicolumn{6}{|c|}{ Compounds ( $\mu \mathrm{M})$} \\
\hline & Anthranilate & Tyrosine & $p$-Coumarate & Caffeate & Avn D & Avn F \\
\hline pAvnDF1 & $1498 \pm 76$ & $6.0 \pm 1.0$ & nd & $0.28 \pm 0.07$ & nd & nd \\
\hline $\mathrm{pSO}+\mathrm{pY}+\mathrm{pAvnDF1}$ & $5802 \pm 298$ & $6286 \pm 150$ & nd & $65.1 \pm 8.3$ & $0.07 \pm 0.00$ & $0.11 \pm 0.04$ \\
\hline pAvnDF2 & $1521 \pm 44$ & $2.0 \pm 0.2$ & $0.10 \pm 0.01$ & $0.13 \pm 0.03$ & $0.03 \pm 0.00$ & nd \\
\hline$p S 0+p Y+p A v n D F 2$ & $5644 \pm 288$ & $2503 \pm 313$ & $11.9 \pm 2.2$ & $14.9 \pm 1.6$ & $4.1 \pm 0.7$ & $0.54 \pm 0.16$ \\
\hline
\end{tabular}

Values are the means \pm SD of five independent clones. nd not detected. 


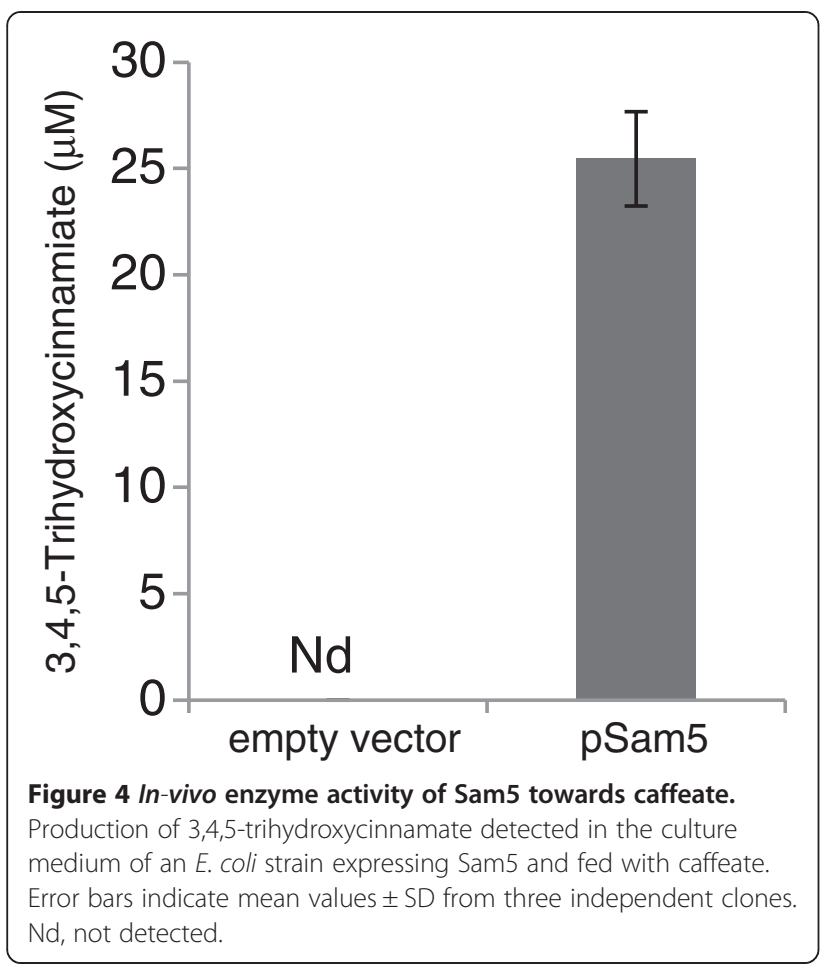

acyltransferase HCBT allowed the exclusive biological synthesis of cinnamoyl anthranilates. For instance, no mass peaks corresponding to other phenylpropenoyl-amino acid amides consisting of a tryptophan, tyrosine or an Ldopa moiety - nor to hydroxycinnamate esters of shikimate or quinate - could be detected in the culture medium of our different E. coli Avn-producing strains.

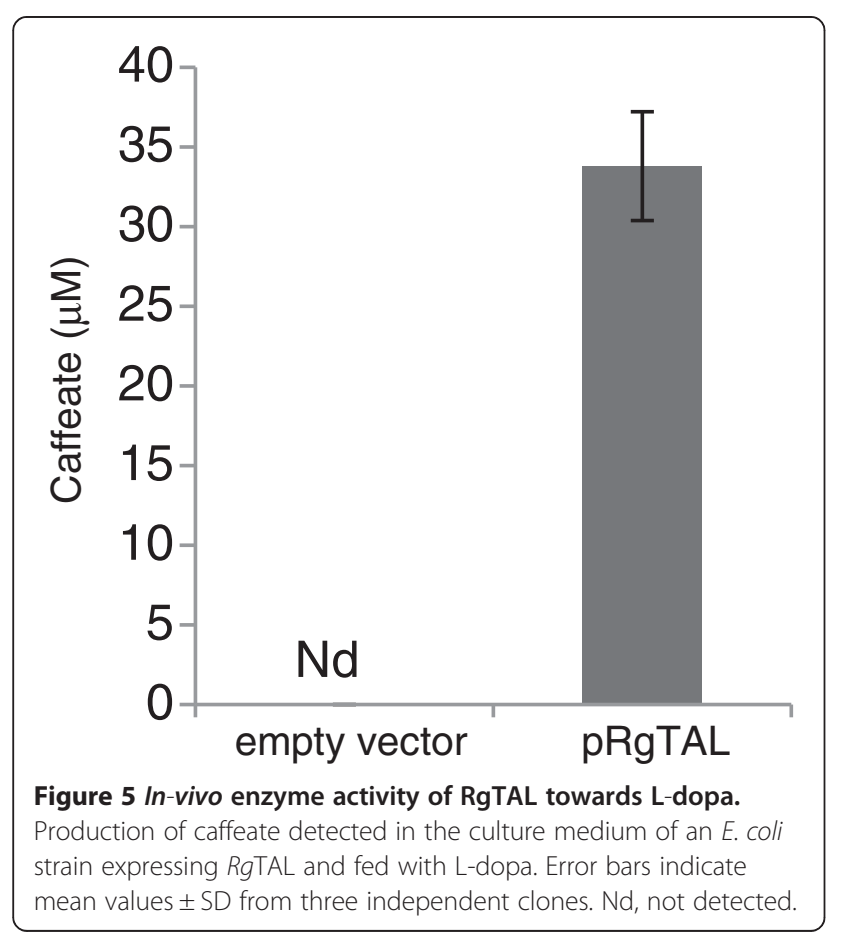

The discovery that $R g$ TAL has L-dopa ammonia-lyase (DAL) activity is of interest and provides some opportunities for the design of new enzymes with a higher DAL/ TAL activity ratio. In combination with tyrosine hydroxylase complexes such as $\mathrm{HpaBC}$, such engineered DALs could be used to improve the bioproduction of caffeate from tyrosine via L-dopa and without generating $p$ coumarate as an intermediate, a competitive precursor for the biosynthesis of Avn F. Furthermore, the impact of expressing in our system 4CLs other than Nt4CL1 should be considered; especially in regard to production of Avn F, because Nt4CL1 is known to be less active with caffeate as a substrate compared to $p$-coumarate [56,57]. Finally, our rationally designed pathway can serve as a framework for improvement of Avn production using combinatorial approaches that have been shown previously to increase tyrosine production [58]. As an adjunct to the recent development of procedures that use safe methylating agents [59], this study describes a basis for eco-friendly production of cinnamoyl anthranilates such as Avn D and Avn F and can serve as a scaffold for the synthesis of more elaborate molecules such as tranilast and its analogs.

\section{Methods}

\section{Chemicals and enzymes}

The following chemicals and enzymes were used in this study: $p$-coumarate, L-tyrosine, anthranilate, L-dopa, isopropyl- $\beta$-D-thiogalactopyranoside (IPTG) (Sigma-Aldrich, St. Louis, MO, USA), caffeate (MPBiomedicals, Solon, OH, USA), 3,4,5-trihydroxycinnamate (Apin Chemicals Ltd, Abingdon, UK), restriction enzymes (NEB, Ipswich, MA, USA), PhusionHigh-Fidelity DNA Polymerase (Thermo Scientific, Waltham, MA, USA), Rapid DNA ligase Kit (Roche Applied Science, Indianapolis, IN, USA). All the enzymes were used in accordance with instructions provided by the manufacturers. $N-\left(4^{\prime}-\right.$ hydroxy-(E)-cinnamoyl)-anthranilate (Avn D) and $N-\left(3^{\prime}, 4^{\prime}-\right.$ dihydroxy-(E)-cinnamoyl)-anthranilate (Avn F) were prepared as described $[1,38]$.

\section{Strains, plasmids, media, and growth conditions}

E. coli DH10B (Life technologies, Foster City, CA, USA) was used for gene cloning and plasmid propagation. Bacterial strains and plasmids used in this study are described in Table 3. E. coli strain W3110 trpD9923 was obtained from the E. coli Genetic Stock Center (Yale University, New Haven, CT). E. coli cells for gene cloning and plasmid propagation were grown in Luria-Bertani (LB) medium at $37^{\circ} \mathrm{C}$. For cinnamoyl anthranilate production, E. coli W3110 trpD9923 was used and cultured at $37^{\circ} \mathrm{C}$ in MOPS (morpholinepropanesulfonic acid)-M9 minimal medium [60] containing $1 \%$ glucose, $10 \mu \mathrm{g} / \mathrm{mL}$ vitamin B1, $20 \mu \mathrm{g} / \mathrm{mL}$ tryptophan, and supplemented with the appropriate amounts of antibiotics: carbenicillin $(100 \mu \mathrm{g} / \mathrm{ml})$, 
chloramphenicol (30 $\mu \mathrm{g} / \mathrm{ml})$, and/or kanamycin $(50 \mu \mathrm{g} / \mathrm{ml})$. Independent clones were first streaked onto solid MOPSM9 minimal medium. 10-ml cultures in flasks were started at $\mathrm{OD}_{600}=0.05$ from overnight cultures, and induced eight hours later by addition of IPTG to a final concentration of $0.1 \mathrm{mM}$. For feeding experiments, $300 \mu \mathrm{M} p$-coumarate or caffeate was added to the medium at the time of induction. Samples used to analyze tyrosine, anthranilate, $p$ coumarate, caffeate, L-dopa, 3,4,5-trihydroxycinnamateand cinnamoyl anthranilates content were collected after 24 hours of culture. E. coli BL21(DE3) was used and cultured at $37^{\circ} \mathrm{C}$ in MOPS-M9 minimal medium containing $1 \%$ glucose and carbenicillin $(100 \mu \mathrm{g} / \mathrm{ml})$ or kanamycin $(50 \mu \mathrm{g} / \mathrm{ml})$ for in-vivo enzyme activities. 10-ml cultures in flasks were started at $\mathrm{OD}_{600}=0.05$ from overnight cultures of clones containing pSam 5 or pRgTAL, and induced and fed 5 hours later by addition of IPTG $(0.1 \mathrm{mM})$ and caffeate $(200 \mu \mathrm{M})$ or L-dopa $(100 \mu \mathrm{M})$, respectively. Samples used to analyze 3,4,5-trihydroxycinnamate and caffeate were collected after 24 hours of culture.

\section{Construction of plasmids}

The BglBricks cloning strategy and the BglBricks vectors $[62,63]$ were used for gene assembly. All the forward primers consist of a BglII restriction site at the $5^{\prime}$-end, followed by the Shine-Dalgarno sequence prior to the start codon. The reverse primers consist of the XhoI and BamHI restriction sites at the $5^{\prime}$-end. For the pAvn construct, the gene sequence encoding HCBT (GenBank: CAB06427) from [38] was amplified using the primers HCBTfw and HCBTrv listed in Additional file 2: Table S1. The PCR product was digested with BglII / XhoI and ligated into the $\mathrm{pBbA5c}$ plasmid [56] between theBamHI and XhoI restriction sites. The cDNA clone corresponding to Nt4CL1 (GenBank: U50845) from [48] was amplified using the primers 4CLfw and 4CLrv (Additional file 2: Table S1), digested with BglII / XhoI and ligated into the pBbA5c::HCBT construct previously digested with BamHI / XhoI to yield the pAvn plasmid.

For the construction of pAvnD, a gene sequence encoding $\operatorname{RgTAL}$ (GenBank: AAA33883) was synthesized (Genescript, NJ, USA) and amplified using the primers TALfw and TALrv listed in Additional file 2: Table S1. The PCR product was digested with BglII / XhoI and ligated downstream Nt4CL1 into pAvn previously digested with BamHI / XhoI. The $\operatorname{Rg} \mathrm{TAL}$ gene sequence was also ligated downstream the T7 promoter into the pBbE7k plasmid [62] between the BamHI and XhoI sites to obtain the pRgTAL construct.

Table 3 Plasmids and strains used in this study

\begin{tabular}{|c|c|c|}
\hline Plasmid or Strain & Description & References \\
\hline \multicolumn{3}{|l|}{ Base plasmids } \\
\hline pZS21 & pSC101; Kan' $P_{\text {LtetO-1 }}$ & {$[61]$} \\
\hline pBbB5a & pBBR1; Ampr/acl Placuv5 & [62] \\
\hline $\mathrm{pBbA5c}$ & p15A; Cmrlacl Placuv5 & [62] \\
\hline pBbE1a & colE1; Amp ${ }^{r} / a c P_{\text {trc }}$ & [62] \\
\hline pBbE7k & colE1; Kannlacl $\mathrm{P}_{\mathrm{T} 7}$ & {$[62]$} \\
\hline \multicolumn{3}{|l|}{ Shikimate plasmid } \\
\hline pso & pZS21:.ydiB-aroD-aroB-aroG*-ppsA-tktA & [45] \\
\hline \multicolumn{3}{|l|}{ Tyrosine plasmid } \\
\hline pY & pBbB5a::tyrB-tyrA*-aroC-aroA-aroL & This study \\
\hline \multicolumn{3}{|c|}{ Cinnamoyl anthranilates plasmids } \\
\hline pAvn & $\mathrm{pBbA5c::HCBT-4CL1}$ & This study \\
\hline pAvnD & pBbA5c::HCBT-4CL1-tal & This study \\
\hline pAvnDF1 & pBbA5c::HCBT-4CL1-tal-P trc-sam5 $_{1}$ & This study \\
\hline pAvnDF2 & pBbA5c::HCBT-4CL1-tal-P trc - hpaB-hpaC & This study \\
\hline \multicolumn{3}{|l|}{ Other plasmids } \\
\hline pSam5 & pBbE1a::sam5 & This study \\
\hline pRgTAL & pBbE7k::tal & This study \\
\hline \multicolumn{3}{|l|}{ Strains } \\
\hline W3110 trpD9923 & $\begin{array}{l}\text { W3110 [F- } \lambda \text { - INV (rrnD-rrnE) 1] tryptophan auxotroph, randomly } \\
\text { mutagenized by treatment with ultraviolet radiation }\end{array}$ & {$[41,42]$} \\
\hline $\mathrm{DH} 10 \mathrm{~B}$ & Cloning host & Life technologies \\
\hline BL21(DE3) & Expression host & Life technologies \\
\hline
\end{tabular}


For the pAvnDF1 construct, a gene sequence encoding Sam5 (GenBank: ABC88666.1) was synthesized (Genescript, NJ, USA) with the BglBricks restriction sites EcoRI and BgIII followed by the Shine-Dalgarno sequence at the $5^{\prime}$-end, and with BamHI and XhoI restriction sites at the $3{ }^{\prime}$-end. The sam5 fragment was released by BglII / XhoI digestions and cloned between the BamHI and XhoI sites of the pBbE1a plasmid [63], downstream the terminator - promoter combination sequence $\mathrm{T} 1-\mathrm{P}_{\text {trc }}$, to yield the pSam5 plasmid. The T1-P trc $^{-S a m} 5$ fragment was released from pSam5 with BglII / XhoI digestions and ligated downstream RgTAL into pAvnD previously digested with BamHI and XhoI.

For the pAvnDF2 construct, the $h p a B C$ operon, which encodes HpaB (GenBank: CAQ34705) and HpaC (GenBank: CAQ34704) was amplified from E. coli BL21 (DE3) genomic DNA using primers hpaBCfw and hpaBCrv (Additional file 2: Table S1). The PCR product was ligated into the pCR-4BluntTOPO vector (Life technologies, Foster City, CA, USA) and a sequenced-verified clone was cured by site-directed mutagenesis to remove an internal BglII restriction site (nucleotides 83-88) using the primers SDM-BglIIfw and SDM-BglIIrv (Additional file 2: Table S1). The cured hpaBC operon was cloned into the pBbE1a plasmid downstream the T1- $\mathrm{P}_{\text {trc }}$ sequence. The T1- $\mathrm{P}_{\text {trc }}-h p a B C$ fragment was released with BglII / XhoI digestions and ligated downstream $\mathrm{RgTAL}$ into pAvnD previously digested with BamHI and XhoI. For the construction of the pY plasmid, the tyrosine operon in the pY1 plasmid [45] was released with BglII / XhoI digestions and cloned into the pBbB5a plasmid between the BamHI and XhoI restriction sites.

LC-MS analysis of cinnamoyl anthranilates and precursors All metabolites were quantified using HPLC-electrospray ionization (ESI)-time-of-flight (TOF) MS. An aliquot of the culture medium was cleared by centrifugation (21,000xg, $\left.5 \mathrm{~min}, 4^{\circ} \mathrm{C}\right)$, mixed with an equal volume of cold methanol-water (1:1, v/v), and filtered using Amicon Ultra centrifugal filters (3,000 Da MW cut off regenerated cellulose membrane; Millipore, Billerica, MA) prior to analysis. For the quantification of intracellular Avn, a cell pellet from $5 \mathrm{ml}$ of culture was washed three times with water, suspended in cold methanol-water $(1: 1, \mathrm{v} / \mathrm{v})$, sonicated twice for $30 \mathrm{~s}$ and centrifuged $(21,000 \mathrm{xg}, 5 \mathrm{~min}$, $4^{\circ} \mathrm{C}$ ). The supernatant was collected and filtered before analysis. The separation of metabolites was conducted on the fermentation-monitoring HPX-87H column with $8 \%$ cross-linkage (150-mm length, 7.8-mm inside diameter, and 9- $\mu \mathrm{m}$ particle size; Bio-Rad, Richmond, CA) using an Agilent Technologies 1100 Series HPLC system. A sample injection volume of $10 \mu \mathrm{l}$ was used throughout. The sample tray and column compartment were set to 4 and $50^{\circ} \mathrm{C}$, respectively. Metabolites were eluted isocratically with a mobile-phase composition of $0.1 \%$ formic acid in water at a flow rate of $0.5 \mathrm{ml} / \mathrm{min}$. The HPLC system was coupled to an Agilent Technologies 6210 series time-of-flight mass spectrometer (for LC-TOF MS) via a MassHunter workstation (Agilent Technologies, CA). Drying and nebulizing gases were set to 13 liters/min and $30 \mathrm{lb} /$ $i^{2}$, respectively, and a drying-gas temperature of $330^{\circ} \mathrm{C}$ was used throughout. ESI was conducted in the negative ion mode and a capillary voltage of $-3,500 \mathrm{~V}$ was utilized. All other MS conditions were described previously [38]. Metabolites were quantified via seven-point calibration curves of authentic standard compounds for which the $R^{2}$ coefficients were $\geq 0.99$.

\section{Additional files}

Additional file 1: Figure S1. LC-TOF MS analysis of 3,4,5-

trihydroxycinnamate produced by engineered $E$. coli. (A) A sample from the medium of the strain harboring pAvnDF1 after 24 hours of culture. (B) Standard, $25 \mu \mathrm{M}$ 3,4,5-trihydroxycinnamate solution.

Additional file 2: Table S1. Primers used in this study.

\section{Abbreviations}

Avn: Avenanthramide; DHAvnD: Dihydroavenanthramide D; 4CL:

4-Coumarate/CoA ligase; HCBT: Hydroxycinnamoyl/benzoyl-CoA/anthranilate $\mathrm{N}$-hydroxycinnamoyl/benzoyltransferase; TAL: Tyrosine ammonia lyase; CoA: Coenzyme A.

\section{Competing interests}

JDK has financial conflicts of interest in Amyris, LS9, and Lygos. DL has financial conflicts of interest in Afingen.

\section{Authors' contributions}

All authors wrote the manuscript. AE and DJ performed the experiments. EEKB conducted the LC-MS analyses. FWC synthesized the Avn D and Avn F standards. DL and JDK supervised the research. All authors read and approved the final version of the manuscript.

\section{Acknowledgements}

Authors are thankful to Dr. Carsten Rautengarten for providing the Nt4CL1 cDNA clone and Sabin Russell for language editing of the manuscript. This work was part of the DOE Joint BioEnergy Institute (http://www.jbei.org) supported by the U. S. Department of Energy, Office of Science,

Office of Biological and Environmental Research, through contract DE-AC02-05CH11231 between Lawrence Berkeley National Laboratory and the U.S. Department of Energy.

\section{Author details}

${ }^{1}$ Joint BioEnergy Institute, Emeryville, CA 94608, USA. ${ }^{2}$ Physical Biosciences Division, Lawrence Berkeley National Laboratory, Berkeley, CA 94720, USA. ${ }^{3}$ California Institute for Quantitative Biosciences and the Synthetic Biology Institute at UC Berkeley, Berkeley, CA 94720, USA. ${ }^{4}$ Eastern Cereal and Oilseed Research Centre, Agriculture and Agri-Food, Ottawa, ON K1A 0C5, Canada. ${ }^{5}$ Department of Bioengineering, Department of Chemical \& Biomolecular Engineering, University of California, Berkeley, CA 94720, USA.

Received: 25 May 2013 Accepted: 18 June 2013

Published: 28 June 2013

\section{References}

1. Collins FW: Oat phenolics: avenanthramides, novel substituted Ncinnamoylanthranilate alkaloids from oat groats and hulls. J Agric Food Chem 1989, 37:60-66. 
2. Collins FW, Mullin WJ: High-performance liquid chromatographic determination of avenanthramides, $\mathrm{N}$-aroylanthranilic acid alkaloids from oats. J Chromatogr 1988, 45:363-370.

3. Peterson DM, Burrup DE, Wesenberg DM, Erickson CA: Relationships among agronomic traits and grain composition in Oat genotypes grown in different environments. Crop Sci 2005, 45:1249-1255.

4. Singh R, De S, Belkheir A: Avena sativa (Oat), a potential neutraceutical and therapeutic agent: an overview. Crit Rev Food SciNut 2013, 53:126-144.

5. Meydani M: Potential health benefits of avenanthramides of oats. Nutr Rev 2009, 67:731-735.

6. Guo W, Kong E, Meydani M: Dietary polyphenols, inflammation, and cancer. Nutr Cancer 2009, 61:807-810.

7. Ji LL, Lay D, Chung E, Fu Y, Peterson DM: Effect of avenathramides on oxidant generation and antioxidant enzyme activity in exercised rats. Nutr Res 2003, 23:1579-1590.

8. O'Moore KM, Vanlandschoot CM, Dickman JR, Figi AR, Rothert AM, Ji LL: Effect of avenanthramides on rat skeletal muscle injury induced by lengthening contraction. Med Sci Sports Exer 2005, 37(Suppl 5):466.

9. Ren Y, Yang X, Niu X, Liu S, Ren G: Chemical characterization of the avenanthramide-rich extract from oat and its effect on D-galactose -induced oxidative stress in mice. J Agric Food Chem 2011, 59:206-211.

10. Liu L, Zubik L, Collins FW, Marko M, Meydani M: The antiatherogenic potential of oat phenolic compounds. Atherosclerosis 2004, 175:39-49.

11. Guo W, Wise ML, Collins FW, Meydani M: Avenanthramides, polyphenols from oats, inhibit IL-1 beta-induced NF-kappaB activation in endothelial cells. Free RadicBiol Med 2008, 44:415-429.

12. LV N, Song MY, Lee YR, Choi HN, Kwon KB, Park JW, Park BH: Dihydroavenanthramide D protects pancreatic beta-cells from cytokine and streptozotocin toxicity. Biochem Biophys Res Commun 2009, 387:97-102.

13. Lee YR, Noh EM, Oh HJ, Hur H, Kim JM, Han JH, Hwang JK, Park BH, Park JW, Youn HJ, Jung SH, Kim BS, Jung JY, Lee SH, Park CS, Kim JS: Dihydroavenanthramide D inhibits human breast cancer cell invasion through suppression of MMP-9 expression. Biochem Biophys Res Commun 2011, 405:552-557.

14. Sur R, Nigam A, Grote D, Liebel F, Southall MD: Avenanthramides, polyphenols from oats, exhibit anti-inflammatory and anti-itch activity. Arch Dermatol Res 2008, 300:569-574.

15. Heuschkel S, Wohlrab J, Schmaus G, Neubert RH: Modulation of dihydroavenanthramide $D$ release and skin penetration by 1,2alkanediols. Eur J Pharm Biopharm 2008, 70:239-247.

16. Nie L, Wise ML, Peterson DM, Meydani M: Avenanthramide, a polyphenol from oats, inhibits vascular smooth muscle cell proliferation and enhances nitric oxide production. Atherosclerosis 2006, 186:260-266.

17. Nie L, Wise M, Peterson D, Meydani M: Mechanism by which avenanthramide-c, a polyphenol of oats, blocks cell cycle progression in vascular smooth muscle cells. Free Radic Biol Med 2006, 41:702-708.

18. Guo W, Nie L, Wu D, Wise ML, Collins FW, Meydani SN, Meydani M: Avenanthramides inhibit proliferation of human colon cancer cell lines in vitro. Nutr Cancer 2010, 62:1007-1016.

19. Azuma H, Banno K, Yoshimura T: Pharmacological properties of N-(3',4'dimethoxycinnamoyl) anthranilic acid (N-5'), a new anti-atopic agent. $\mathrm{Br}$ J Pharmacol 1976, 58:483-488.

20. Isaji M, Miyata H, Ajisawa Y: Tranilast: a new application in the cardiovascular field as an antiproliferative drug. Cardiovasc Drug Rev 1998, 16:288-299.

21. Suzawa H, Kikuchi S, Ichikawa K, Koda A: Inhibitory action of Tranilast, an anti-allergic drug, on the release of cytokines and PGE2 from human monocytes-macrophages. Jpn J Pharmacol 1992, 60:85-90.

22. Crittenden DB, Pillinger MH: The year in gout - 2010-2011. Bull NYU Hosp Jt Dis 2011, 69:257-263.

23. Inglis JJ, Criado G, Andrews M, Feldmann M, Williams RO, Selley ML: The anti-allergic drug, N-(3',4'-dimethoxycinnamonyl) anthranilic acid, exhibits potent anti-inflammatory and analgesic properties in arthritis. Rheumatology 2007, 46:1428-1432.

24. Platten M, Ho PP, Youssef S, Fontoura P, Garren H, Hur EM, Gupta R, Lee LY, Kidd BA, Robinson WH, Sobel RA, Selley ML, Steinman L: Treatment of autoimmune neuroinflammation with a synthetic tryptophan metabolite. Science 2005, 310:850-855.

25. Rogosnitzky M, Danks R, Kardash E: Therapeutic Potential of Tranilast, an Anti-allergy Drug, in Proliferative Disorders. Anticancer Res 2012 , 32:2471-2478.
26. Zammit SC, Cox AJ, Gow RM, Zhang Y, Gilbert RE, Krum H, Kelly DJ, Williams SJ: Evaluation and optimization of antifibrotic activity of cinnamoyl anthranilates. Bioorg Med Chem Lett 2009, 19:7003-7006.

27. Zhang Y, Edgley AJ, Cox AJ, Powell AK, Wang B, Kompa AR, Stapleton DI, Zammit SC, Williams SJ, Krum H, Gilbert RE, Kelly DJ: FT011, a new antifibrotic drug, attenuates fibrosis and chronic heart failure in experimental diabetic cardiomyopathy. Eur J Heart Fail 2012, 14:549-562.

28. Zhang Y, Elsik M, Edgley AJ, Cox AJ, Kompa AR, Wang B, Tan CY, Khong FL, Stapleton DI, Zammit S, Williams SJ, Gilbert RE, Krum H, Kelly DJ: A new anti-fibrotic drug attenuates cardiac remodeling and systolic dysfunction following experimental myocardial infarction. Int J Cardio 2012. doi:10.1016/j.icard.2012.11.067. PMID:23219315.

29. Tan SM, Zhang Y, Wang B, Tan CY, Zammit SC, Williams SJ, Krum H, Kelly DJ: An orally active anti-fibrotic compound, FT23, attenuates structural and functional abnormalities in an experimental model of diabetic cardiomyopathy. Clin Exp Pharmacol Physiol 2012, 39:650-656.

30. Gilbert RE, Zhang Y, Williams SJ, Zammit SC, Stapleton DI, Cox AJ, Krum H, Langham R, Kelly DJ: A purpose-synthesised anti-fibrotic agent attenuates experimental kidney diseases in the rat. PLoS One 2012, 7:e47160.

31. Keasling JD: Manufacturing molecules through metabolic engineering. Science 2010, 330:1355-1358.

32. Pickens $L B$, Tang $Y$, Chooi $Y H$ : Metabolic engineering for the production of natural products. Annu Rev Chem Biomol Eng 2011, 2:211-236.

33. Williams SJ, Stapleton D, Zammit S, James D, Ernest R, Krum H: Therapeutic compounds. United States: Patent Application No. 2010/0130497 A1; 2010.

34. Fielder DA, Redmond MJ, Cottrell IW: Avenanthramide-containing compositions. United States: Patent Application No. 2010/0267662 A1; 2010

35. Yang Q, Reinhard K, Schiltz E, Matern U: Characterization and heterologous expression of hydroxycinnamoyl/benzoyl-CoA:anthranilate $\mathrm{N}$-hydroxycinnamoyl/benzoyltransferase from elicited cell cultures of carnation Dianthus caryophyllus L. Plant Mol Biol 1997, 35:777-789.

36. D'Auria J: Acyltransferases in plants: a good time to be BAHD. Curr Opin Plant Biol 2006, 9:331-340.

37. Hamberger B, Hahlbrock K: The 4-coumarate:CoA ligase gene family in Arabidopsis thaliana comprises one rare, sinapate-activating and three commonly occurring isoenzymes. Proc Natl Acad Sci USA 2004, 101:2209-2214.

38. Eudes A, Baidoo EE, Yang F, Burd H, Hadi MZ, Collins FW, Keasling JD, Loqué D: Production of tranilast [N-(3',4'-dimethoxycinnamoyl)anthranilic acid] and its analogs in yeast Saccharomyces cerevisiae. Appl Microbiol Biotechnol 2011, 89:989-1000.

39. Ajikumar PK, Tyo K, Carlsen S, Mucha O, Phon TH, Stephanopoulos G: Terpenoids: opportunities for biosynthesis of natural product drugs using engineered microorganisms. Mol Pharm 2008, 5:167-190.

40. Gosset G: Production of aromatic compounds in bacteria. Curr Opin Biotechnol 2009, 20:651-658.

41. Balderas-Hernández VE, Sabido-Ramos A, Silva P, Cabrera-Valladares N, Hernández-Chávez G, Báez-Viveros JL, Martínez A, Bolívar F, Gosset G: Metabolic engineering for improving anthranilate synthesis from glucose in Escherichia coli. Microb Cell Fact 2009, 8:19.

42. Yanofsky C, Horn V, Bonner M, Stasiowski S: Polarity and enzyme functions in mutants of the first three genes of the tryptophan operon of Escherichia col. Genetics 1971, 69:409-433.

43. Vannelli T, Wei Qi W, Sweigard J, Gatenby AA, Sariaslani FS: Production of p-hydroxycinnamic acid from glucose in Saccharomyces cerevisiae and Escherichia coli by expression of heterologous genes from plants and fungi. Metab Eng 2007, 9:142-151.

44. Qi WW, Vannelli T, Breinig S, Ben-Bassat A, Gatenby AA, Haynie SL, Sariaslani FS: Functional expression of prokaryotic and eukaryotic genes in Escherichia coli for conversion of glucose to p-hydroxystyrene. Metab Eng 2007, 9:268-276.

45. Juminaga D, Baidoo EE, Redding-Johanson AM, Batth TS, Burd H, Mukhopadhyay A, Petzold CJ, Keasling JD: Modular engineering of L-tyrosine production in Escherichia coli. App/ Environ Microbiol 2012, 78:89-98.

46. Lin Y, Yan Y: Biosynthesis of caffeic acid in Escherichia coli using its endogenous hydroxylase complex. Microb Cell Fact 2012, 11:42.

47. Berner M, Krug D, Bihlmaier C, Vente A, Müller R, Bechthold A: Genes and enzymes involved in caffeic acid biosynthesis in the actinomyceteSaccharothrix espanaensis. J Bacteriol 2006, 188:2666-2673. 
48. Rautengarten C, Baidoo E, Keasling JD, Scheller HV: A simple method for enzymatic synthesis of unlabeled and radiolabeled hydroxycinnamateCoA. Bioenerg Res 2010, 3:115-122.

49. Kang SY, Choi O, Lee JK, Hwang BY, Uhm TB, Hong YS: Artificial biosynthesis of phenylpropanoic acids in a tyrosine overproducing Escherichia coli strain. Microb Cell Fact 2012, 11:153.

50. Zhang H, Stephanopoulos G: Engineering E. coli for caffeic acid biosynthesis from renewable sugars. Appl Microbiol Biotechnol 2012, 97:3333.

51. Choi O, Wu CZ, Kang SY, Ahn JS, Uhm TB, Hong YS: Biosynthesis of plantspecific phenylpropanoids by construction of an artificial biosynthetic pathway in Escherichia coli. J Ind Microbiol Biotechnol 2011, 38:1657-1665.

52. Muñoz AJ, Hernández-Chávez G, De Anda R, Martínez A, Bolívar F, Gosset G: Metabolic engineering of Escherichia coli for improving L-3,4dihydroxyphenylalanine (L-DOPA) synthesis from glucose. J Ind Microbiol Biotechnol 2011, 38:1845-1852.

53. Lee J-Y, Xun L: Novel biological process for I-dopa production from I-tyrosine by $p$-hydroxyphenylacetate 3-hydroxylase. Biotechnol Lett 1998, 20:479-482.

54. Noel JP, Louie GV, Bowman ME, Moore BS, Moffitt MC: Substrate switched ammonia lyases and mutases. United States: Patent Application No. 2009/ 0011400 A1; 2009

55. Fowler ZL, Gikandi WW, Koffas MAG: Increased malonylcoenzyme A biosynthesis by tuning the Escherichia coli metabolic network and its Application to flavanone production. Appl Environ Microbiol 2009, 75:5831-5839.

56. Lee D, Douglas CJ: Two divergent members of a tobacco 4-coumarate: coenzyme A ligase $(4 \mathrm{CL})$ gene family. $C D N A$ structure, gene inheritance and expression, and properties of recombinant proteins. Plant Physiol 1996, 112:193-205.

57. Yun MS, Chen W, Deng F, Kiyokawa T, Mametsuka K, Yogo Y: An in vitro screening assay to discover novel inhibitors of 4-coumarate:CoA ligase. Pest Manag Sci 2006, 62:1065-1071.

58. Santos CN, Xiao W, Stephanopoulos G: Rational, combinatorial, and genomic approaches for engineering L-tyrosine production in Escherichia coli. Proc Natl Acad Sci USA 2012, 109:13538-13543.

59. Bernini $R$, Crisante F, Ginnasi MC: A convenient and safe O-methylation of flavonoids with dimethyl carbonate (DMC). Molecules 2011, 16:1418-1425.

60. Neidhardt FC, Bloch PL, Smith DF: Culture medium for enterobacteria. J Bacteriol 1974, 119:736-747.

61. Lutz R, Bujard $\mathrm{H}$ : Independent and tight regulation of transcriptional units in Escherichia coli via the LacR/O, the TetR/O and AraC/I1-I2 regulatory elements. Nucleic Acids Res 1997, 25:1203-1210.

62. Lee TS, Krupa RA, Zhang F, Hajimorad M, Holtz WJ, Prasad N, Lee SK, Keasling JD: BglBrick vectors and datasheets: a synthetic biology platform for gene expression. J BiolEng 2011, 5:12.

63. Anderson JC, Dueber JE, Leguia M, Wu GC, Goler JA, Arkin AP, Keasling JD: BglBricks: a flexible standard for biological part assembly. J Biol Eng 2010, 4:1.

doi:10.1186/1475-2859-12-62

Cite this article as: Eudes et al:: Production of hydroxycinnamoyl anthranilates from glucose in Escherichia coli. Microbial Cell Factories 2013 12:62.

\section{Submit your next manuscript to BioMed Central and take full advantage of:}

- Convenient online submission

- Thorough peer review

- No space constraints or color figure charges

- Immediate publication on acceptance

- Inclusion in PubMed, CAS, Scopus and Google Scholar

- Research which is freely available for redistribution 